\title{
Fixed Point Results on Multiplicative Semi-Metric Space
}

\author{
B. Vijayabaskerreddy ${ }^{1 *}$, V. Srinivas ${ }^{2}$ \\ ${ }^{1}$ Department of Mathematics, Sreenidhi Institute of Science and Technology, Hyderabad, India \\ ${ }^{2}$ Department of Mathematics, University College, Saifabad, Osmania University, Hyderabad, India
}

Received 31 December 2019, accepted in final revised form 23 March 2020

\begin{abstract}
In this paper we introduce the notion of the Multiplicative Semi-Metric Space and proved common fixed point theorems. We establish fixed point theorems for four self-maps which can be extended to derive common fixed point theorems involving any finite number of mappings in Multiplicative Semi Metric Space. Further examples are discussed to show that compatible mappings of type-E, weakly compatible mappings and reciprocally-continuous mappings are weaker forms of compatible mappings and continuous mappings respectively. The main objective of this article is to prove the unique common fixed point theorems and employing the notion of the compatible mappings of type-E, reciprocally-continuous mappings in the Multiplicative Semi Metric Space. Our result generalizes the concept of Multiplicative Metric Space as it does not involve the multiplicative triangle inequality.
\end{abstract}

Keywords: Fixed point; Compatible mappings of type-E; Reciprocally-Continuous; Weaklyreciprocally-continuous; Multiplicative Semi Metric Space.

() 2020 JSR Publications. ISSN: 2070-0237 (Print); 2070-0245 (Online). All rights reserved. doi: http://dx.doi.org/10.3329/jsr.v12i3.44754 J. Sci. Res. 12 (3), 341-348 (2020)

\section{Introduction}

In 1906, the idea of metric space was initiated by Frechet, which is an important tool in scientific area particularly in fixed point theory. Since then metric space has been generalized in several ways as quasi metric space, semi metric space etc.

In the $17^{\text {th }}$ century, Newton and Leibnitz created most important mathematical concepts in differential and integral calculus. In the year 1972, Grossman and Katz [1] gave multiplicative calculus also known as Non-Newtonian calculus. In the year 2008, Bashirov et al. [2] initiated the new metric space named as multiplicative metric space. In Non-Newtonian calculus, Ozavsar et al. [3] studied the notion of convergence and gave unique common fixed point results in the multiplicative metric space.

It can be observed that weaker form of metric space known as semi-metric space which is obtained by dropping triangular inequality in a metric space. In 1922 Austrian -

\footnotetext{
*Corresponding author: basker.bonuga@gmail.com
} 
American mathematician Menger [4] initiated notion of semi-metric space is also known as symmetric space. Thereafter Cicchese [5] verified the common fixed point results by introducing the contractive mappings. In 1988, Jungck [6] introduced the compatible mappings by generalizing the notion of commuting mappings. Singh et al. [7] introduced the concept of compatible mappings of type-E and its weaker forms. In 1999, Pant [8] introduced the notion of reciprocally continuous and its weaker forms. In the recent past, many fixed point theorems are being generated $[9,10]$ using the above ideas and also the concept of compatible of type-E is significantly used in Menger spaces [11].

Our main objective is to obtain some results on compatible mappings of type-E and obtain fixed point theorems without continuity and compatible requirements. Further the concept of compatible of type-E is taken in the splitting form namely A-compatible typeE, S-compatible type-E. Similarly the concept of reciprocally continuity can be divided into two weaker forms, A-weakly-reciprocally-continuous mappings and S-weaklyreciprocally-continuous mappings. In the final part of the paper, a fixed point theorem using compatible mapping of type-E with weakly compatible mappings is presented.

\section{Preliminaries}

Now we give some definitions which are useful in proving our results.

Definition 2.1 [2]: Let $X$ be any non-empty set. A mapping $d^{*}: X \times X \rightarrow \mathbb{R}^{+}$satisfying the following conditions:

(MMS-i) $d^{*}(x, y) \geq 1$ for all $x, y \in X$ and $d^{*}(x, y)=1 \Leftrightarrow x=y$

(MMS-ii) $d^{*}(x, y)=d^{*}(y, x) \forall x, y \in X$

(MMS-iii) $d^{*}(x, z) \leq d^{*}(x, y) \cdot d^{*}(y, z) \forall x, y, z \in X$.

Then the pair $\left(X, d^{*}\right)$ is called Multiplicative Metric Space (MMS).

Definition 2.2: Let $X$ be a non-empty set and the mapping $d^{*}: X \times X \rightarrow \mathbb{R}^{+}$then $\left(X, d^{*}\right)$ is said to be a Multiplicative Semi Metric Space (we denote shortly by MSMS) if it satisfies the following conditions:

$\left(\right.$ MSMS-i): $d^{*}(x, y) \geq 1 \forall x, y \in X$ and $d^{*}(x, y)=1 \Leftrightarrow x=y$

(MSMS-ii): $d^{*}(x, y)=d^{*}(y, x), \forall x, y \in X$.

Definition 2.3: Two self-maps A and S of MSMS $\left(X, d^{*}\right)$ are said to be weakly compatible if they commute at coincidence points, i.e if At $=\mathrm{St}$ for some $t \in$ $\mathrm{X}$ implies ASt $=$ SAt.

Definition 2.4: Let $A$ and $S$ be two self-mappings of a MSMS $\left(X, d^{*}\right)$ are said to be reciprocally - continuous ( $(\mathrm{rc})$ if $\quad \lim _{\mathrm{n} \rightarrow \infty} \mathrm{ASx}_{\mathrm{n}}=\mathrm{At}$ and $\lim _{n \rightarrow \infty} S A x_{n}=$ St whenever $\left\{x_{n}\right\}$ in $X$ such that $\lim _{n \rightarrow \infty} A x_{n}=\lim _{n \rightarrow \infty} S x_{n}=t$ for some $t \in X$.

Definition 2.5: Two self-maps A and $S$ of MSMS (X, $\left.\mathrm{d}^{*}\right)$ are said to be weaklyreciprocally - continuous (wrc) if $\lim _{n \rightarrow \infty} A S x_{n}=$ At or $\lim _{n \rightarrow \infty} S A x_{n}=$ St whenever a sequence $\left\{x_{n}\right\}$ in $X$ such that $\lim _{n \rightarrow \infty} A x_{n}=\lim _{n \rightarrow \infty} S x_{n}=t$ for some $t \in X$.

Definition 2.6: Suppose that $A$ and $S$ are two self-maps of a MSMS $\left(X, d^{*}\right)$, then the pair of self-maps $(A, S)$ is said to be $S$ - weakly - reciprocally - continuous $(S-$ wrc) if 
$\lim _{n \rightarrow \infty} S A x_{n}=S t$ whenever a sequence $\left\{x_{n}\right\}$ in $X$ such that $\lim _{n \rightarrow \infty} A x_{n}=$ $\lim _{\mathrm{n} \rightarrow \infty} S \mathrm{x}_{\mathrm{n}}=\mathrm{t}$ for some $\mathrm{t} \in \mathrm{X}$.

Now we give an example.

Example 2.6.1 [9]: Let $X=\left[0, \frac{1}{2}\right]$ on usual metric space with the following mappings.

$$
A(x)=\left\{\begin{array}{ll}
\frac{1}{6} & \text { if } 0 \leq x<\frac{1}{4} \\
\frac{1}{4} & \text { if } \frac{1}{4} \leq x \leq \frac{1}{2}
\end{array} \quad S(x)= \begin{cases}\frac{1}{8} & \text { if } 0 \leq x<\frac{1}{4} \\
\frac{1}{2}-x & \text { if } \frac{1}{4} \leq x \leq \frac{1}{2}\end{cases}\right.
$$

Clearly the pair of mappings $(A, S)$ is $S$ - wrc but not reciprocally - continuous(rc). For this, take a sequence $\left\{x_{n}\right\}=\frac{1}{4}+\frac{1}{n}$ for $n \geq 1$. Then $\lim _{n \rightarrow \infty} A x_{n}=\lim _{n \rightarrow \infty} S x_{n}=\frac{1}{4}$ and $\lim _{\mathrm{n} \rightarrow \infty} \mathrm{ASx}_{\mathrm{n}}=\frac{1}{6}$ also $\lim _{\mathrm{n} \rightarrow \infty} \mathrm{SAx}_{\mathrm{n}}=\frac{1}{4}$.

Definition 2.7: Two self-mappings $A$ and $S$ of $\operatorname{MSMS}\left(X, d^{*}\right)$ are said to be Compatible of type - (E) (Shortly Comp-(E)) if $\lim _{n \rightarrow \infty} S S x_{n}=\lim _{n \rightarrow \infty} S A x_{n}=$ At and $\lim _{n \rightarrow \infty} A A x_{n}=\lim _{n \rightarrow \infty} A S x_{n}=$ St whenever a sequence $\left\{x_{n}\right\}$ in $X$ such that $\lim _{n \rightarrow \infty} A x_{n}=\lim _{n \rightarrow \infty} S x_{n}=t$ for some $t \in X$.

Example 2.7.1 [10]: Let $X=[0,1]$ be endowed with MSMS. Let the mappings A and $S$ as follows

$$
A(x)=\left\{\begin{array}{cc}
x & \text { if } 0 \leq x \leq \frac{1}{2} \\
\frac{1+x}{4} & \text { if } \frac{1}{2} \leq x \leq 1
\end{array} \quad S(x)= \begin{cases}\frac{1}{2} x & \text { if } 0 \leq x \leq \frac{1}{2} \\
\frac{1}{5} & \text { if } \frac{1}{2} \leq x \leq 1\end{cases}\right.
$$

Take the sequence $\left\{x_{n}\right\}=\frac{1}{n}$ for each $n \geq 1$.

Clearly $\lim _{n \rightarrow \infty} A x_{n}=0=\lim _{n \rightarrow \infty} S x_{n}$ and this implies $\lim _{n \rightarrow \infty} A^{2} x_{n}=0=$ $\lim _{n \rightarrow \infty} A S x_{n}=S(0)$ and $\lim _{n \rightarrow \infty} S^{2} x_{n}=\lim _{n \rightarrow \infty} S A x_{n}=A(0)$.

Therefore $(A, S)$ is compatible type $-(E)$.

Definition 2.8: Two self-maps A and $S$ of $\operatorname{MSMS}\left(\mathrm{X}, \mathrm{d}^{*}\right)$ are said to be A - Compatible of type - (E) (shortly A - Comp - (E) ) if $\lim _{n \rightarrow \infty} A A x_{n}=$ $\lim _{n \rightarrow \infty} A S x_{n}=S t$ whenever $\left\{x_{n}\right\}$ in $X$ such that $\lim _{n \rightarrow \infty} A x_{n}=\lim _{n \rightarrow \infty} S x_{n}=$ $t$ for some $t \in X$.

Example 2.8.1 [10]: Let $X=[0,10]$ be endowed with a MSMS. Consider

$$
A(x)=\left\{\begin{array}{ll}
3-x & \text { if } 0 \leq x \leq 2 \\
\frac{1}{2} & \text { if } 2 \leq x \leq 10
\end{array} S(x)= \begin{cases}\frac{5-x}{2} & \text { if } 0 \leq x \leq 2 \\
\frac{1}{2} & \text { if } 2 \leq x \leq 10\end{cases}\right.
$$

Define a sequence $\left\{x_{n}\right\}$ for $n \geq 1$ where $x_{n}=1-\frac{1}{n}$.

Clearly $\lim _{n \rightarrow \infty} A x_{n}=\lim _{n \rightarrow \infty} S x_{n}=2$ this implies $\lim _{n \rightarrow \infty} A^{2} x_{n}=\lim _{n \rightarrow \infty} A S x_{n}=$ $\mathrm{S}(2)=\frac{1}{2}$ and $\lim _{\mathrm{n} \rightarrow \infty} \mathrm{S}^{2} \mathrm{x}_{\mathrm{n}}=\lim _{\mathrm{n} \rightarrow \infty} \mathrm{SAx}=\frac{1}{2}$, but $\mathrm{A}(2)=1$.

Hence the pair $(A, S)$ is only $S-$ Comp $-(E)$. 


\section{Results}

Theorem 3.1: Let $\left(X, d^{*}\right)$ be a multiplicative symmetric space. Suppose A, B, S and T be four self-maps defined on $\left(X, d^{*}\right)$ and also satisfying the following conditions

(i) the pairs $(\mathrm{A}, \mathrm{S})$ and $(\mathrm{B}, \mathrm{T})$ are weakly-reciprocally-continuous (wrc) and compatible mappings of type-(E)

then $(A, S)$ and $(B, T)$ have a coincidence point each.

Further, the self-mappings $\mathrm{A}, \mathrm{B}, \mathrm{S}$ and $\mathrm{T}$ have a unique common fixed point in $\mathrm{X}$ subject to the constraint

(ii) for all $\mathrm{x}, \mathrm{y} \in \mathrm{X}$ then $\exists$ a real number $\lambda \in\left(0, \frac{1}{2}\right)$ such that $d^{*}($ Ax, By $) \leq\left[d^{*}(\text { Sx, Ty }) d^{*}(A x, S x) d^{*}(B y, T y) d^{*}(S x, B y) d^{*}(A x, B y)\right]^{\frac{\lambda}{6}}$.

Proof: From weakly-reciprocally-continuous of first the pair (A, S) $\exists$ a real sequence $\left\{x_{n}\right\}$ in $X$ such that $\lim _{n \rightarrow \infty} A x_{n}=A$ (or) $\lim _{n \rightarrow \infty} S A x_{n}=S t$ whenever $\lim _{n \rightarrow \infty} A x_{n}=$ $\lim _{\mathrm{n} \rightarrow \infty} S \mathrm{x}_{\mathrm{n}}=\mathrm{t}$ for some $\mathrm{t} \in \mathrm{X}$.

Employing compatible of type $-(\mathrm{E})$ for the given first pair $(\mathrm{A}, \mathrm{S})$, we have $\mathrm{SSx}_{\mathrm{n}}=$ $\lim _{n \rightarrow \infty} S A x_{n}=$ At and $\lim _{n \rightarrow \infty} A A x_{n}=\lim _{n \rightarrow \infty} A S x_{n}=$ St for some $t \in X$.

Then $A t=S t$ and this implies $(A, S)$ has a coincidence point $t$. In a similar way, for the weakly-reciprocally-continuous mapping of the pair $(B, T)$, there may be a sequence $\left\{y_{n}\right\}$ in X such that $\lim _{n \rightarrow \infty} B \mathrm{BTy}_{n}=\mathrm{Bw}$ or $\lim _{\mathrm{n} \rightarrow \infty} \mathrm{TBy}_{\mathrm{n}}=$ Tw whenever $\lim _{\mathrm{n} \rightarrow \infty} \mathrm{By}_{\mathrm{n}}=$ $\lim _{\mathrm{n} \rightarrow \infty} \mathrm{Ty}_{\mathrm{n}}=\mathrm{w}$ for some $\mathrm{w} \in \mathrm{X}$.

Also compatible type-(E) of the second pair (B, T) implies that $\lim _{n \rightarrow \infty} \mathrm{TTy}_{n}=$ $\lim _{n \rightarrow \infty} \mathrm{TBy}_{n}=B w$ and $\lim _{n \rightarrow \infty} \mathrm{BBy}_{n}=\lim _{n \rightarrow \infty} \mathrm{BTy}_{n}=\mathrm{Tw}$ for some $w \in X$.

So we have, $B w=T w$, this indicates that the pair $(B, T)$ is having was coincident point.

We claim $A t=B w$. For suppose $A t \neq B w$

now putting $\mathrm{x}=\mathrm{t}$ and $\mathrm{y}=\mathrm{w}$ in the inequality (ii) of Theorem 3.1, then we get

$d^{*}(A t, B w) \leq\left[d^{*}(S t, T w) d^{*}(A t, S t) d^{*}(B w, T w) d^{*}(S t, B w) d^{*}(A t, B w)\right]^{\frac{\lambda}{6}}$

since $A t=S t$ and $B w=T w$ gives

$\mathrm{d}^{*}(A t, B w) \leq\left[\mathrm{d}^{*}(A t, B w) \mathrm{d}^{*}(A t, A t) \mathrm{d}^{*}(B w, B w) \mathrm{d}^{*}(\mathrm{St}, \mathrm{Bw}) \mathrm{d}^{*}(\mathrm{At}, \mathrm{Bw})\right]^{\frac{\lambda}{6}}$

$\mathrm{d}^{*}(A t, B w) \leq\left[\mathrm{d}^{*}(A t, B w) \mathrm{d}^{*}(A t, A t) \mathrm{d}^{*}(B w, B w) d^{*}(A t, B w) d^{*}(A t, B w)\right]^{\frac{\lambda}{6}}$

$\mathrm{d}^{*}(\mathrm{At}, \mathrm{Bw}) \leq\left[\mathrm{d}^{*}(\mathrm{At}, \mathrm{Bw})(1)(1) \mathrm{d}^{*}(\mathrm{At}, \mathrm{Bw}) \mathrm{d}^{*}(\mathrm{At}, \mathrm{Bw})\right]^{\frac{\lambda}{6}}$

$\mathrm{d}^{*}(\mathrm{At}, \mathrm{Bw}) \leq\left[\mathrm{d}^{* 3}(\mathrm{At}, \mathrm{Bw})\right]^{\frac{\lambda}{6}}$

$\mathrm{d}^{*}(\mathrm{At}, \mathrm{Bw}) \leq\left[\mathrm{d}^{*}(\mathrm{At}, \mathrm{Bw})\right]^{\frac{\lambda}{2}}$ this implies At $=$ Bw.

Now we prove $t=B w$. Suppose $B w \neq t$,

substitute $\mathrm{x}=\mathrm{x}_{\mathrm{n}}, \mathrm{y}=\mathrm{w}$ in the inequality (ii) of Theorem 3.1, then we get

$d^{*}\left(A x_{n}, B w\right) \leq\left[d^{*}\left(S x_{n}, T w\right) d^{*}\left(A x_{n}, S x_{n}\right) d^{*}(B w, T w) d^{*}\left(S x_{n}, B w\right) d^{*}\left(A x_{n}, B w\right)\right]^{\frac{\lambda}{6}}$

letting $\mathrm{n} \rightarrow \infty$ and using $\mathrm{Bw}=\mathrm{Tw}$ we get

$d^{*}(t, B w) \leq\left[d^{*}(t, B w) d^{*}(t, t) d^{*}(B w, B w) d^{*}(t, B w) d^{*}(t, B w)\right]^{\frac{\lambda}{6}}$ 
$d^{*}(t, B w) \leq\left[d^{* 3}(t, B w)\right]^{\frac{\lambda}{6}}$

$d^{*}(t, B w) \leq\left[d^{*}(t, B w)\right]^{\frac{\lambda}{2}}$

this implies $\mathrm{t}=\mathrm{Bw}$.

Hence $\mathrm{t}=\mathrm{At}=\mathrm{St}$.

Next we shall prove that $t=w$. For this consider $x=x_{n}$ and $y=y_{n}$ in the inequality (ii) of Theorem 3.1, we get

$d^{*}\left(A x_{n}, B y_{n}\right) \leq\left[d^{*}\left(S x_{n}, T_{n}\right) d^{*}\left(A x_{n}, S x_{n}\right) d^{*}\left(B y_{n}, T y_{n}\right) d^{*}\left(S x_{n}, B y_{n}\right) d^{*}\left(A x_{n}, B y_{n}\right)\right]^{\frac{\lambda}{6}}$

letting $\mathrm{n} \rightarrow \infty$ we get

$d^{*}(t, w) \leq\left[d^{*}(t, w) d^{*}(t, t) d^{*}(w, w) d^{*}(t, w) d^{*}(t, w)\right]^{\frac{\lambda}{6}}$

$d^{*}(t, w) \leq\left[d^{* 3}(t, w)\right]^{\frac{\lambda}{6}}$

$\mathrm{d}^{*}(\mathrm{t}, \mathrm{w}) \leq\left[\mathrm{d}^{*}(\mathrm{t}, \mathrm{w})\right]^{\frac{\lambda}{2}}$

this implies $\mathrm{t}=\mathrm{w}$.

Hence, $\mathrm{At}=\mathrm{Bt}=\mathrm{St}=\mathrm{Tt}=\mathrm{t}$, which shows that $\mathrm{t}$ is a common fixed point of $\mathrm{A}, \mathrm{B}, \mathrm{S}$ and $\mathrm{T}$.

Finally, to show the uniqueness of the fixed point $\mathrm{t}$, let $\mathrm{q}$ may be considered as another common fixed point of the four given maps then, we get $\mathrm{Aq}=\mathrm{Bq}=\mathrm{Sq}=\mathrm{Tq}=\mathrm{q}$.

Write $\mathrm{x}=\mathrm{t}$ and $\mathrm{y}=\mathrm{q}$ in the inequality (ii) of Theorem 3.1, then

$d^{*}(t, q)=d^{*}(A t, B q) \leq\left[d^{*}(S t, T q) d^{*}(A t, S t) d^{*}(B q, T q) d^{*}(S t, B q) d^{*}(A t, B q)\right]^{\frac{\lambda}{6}}$

$d^{*}(t, q) \leq\left[d^{*}(t, q) d^{*}(t, t) d^{*}(q, q) d^{*}(t, q) d^{*}(t, q)\right]^{\frac{\lambda}{6}}$

$d^{*}(t, q) \leq\left[d^{* 3}(t, q)\right]^{\frac{\lambda}{6}}$

$d^{*}(t, q) \leq\left[d^{*}(t, q)\right]^{\frac{\lambda}{2}}$

this implies $\mathrm{t}=\mathrm{q}$.

Hence the given four self-mappings have the unique common fixed point in X.

Now we prove the existence of common fixed point in another theorem by altering some conditions.

Theorem 3.2: Let $A, B, S$ and $T$ be four self-mappings of a symmetric space $\left(X, d^{*}\right)$. Satisfying the conditions

(i) the pair $(\mathrm{A}, \mathrm{S})$ is A-Compatible mapping of type-(E) and A - weakly reciprocally - continuous

and also

(ii) the pair (B, T) is $B$ - compatible mapping of type - (E) and $\mathrm{B}$ - weakly reciprocally - continuous,

then the two pairs $(A, S)$ and $(B, T)$ have a coincidence point each.

Further if the self-maps satisfy the condition

(iii) $d^{*}(A x, B y) \leq\left[d^{*}(S x, T y) d^{*}(A x, S x) d^{*}(B y, T y) d^{*}(S x, B y) d^{*}(A x, B y)\right]^{\frac{\lambda}{6}}$

$\forall \mathrm{x}, \mathrm{y} \in \mathrm{X}, \lambda \in\left(0, \frac{1}{2}\right)$.

Then the self-maps A, B, S and T are having a unique common fixed point. 
Proof: Since the pair of mappings(A,S) is A-weakly reciprocally-continuous then $\exists$ a sequence $\left\{x_{n}\right\}$ in $X$ such that $\lim _{n \rightarrow \infty} A S x_{n}=$ At whenever $\lim _{n \rightarrow \infty} A x_{n}=\lim _{n \rightarrow \infty} S x_{n}$ for some point $t \in X$.

Applying the condition A-compatible mapping of type-(E) of the pair (A,S) implies $\lim _{n \rightarrow \infty} \mathrm{AAx}_{\mathrm{n}}=\lim _{\mathrm{n} \rightarrow \infty} \mathrm{ASx}_{\mathrm{n}}=$ St for some $\mathrm{t} \in \mathrm{X}$.

Then $A t=S t$, this shows that $t$ is a coincidence point the of $(A, S)$.

Now by the B-weakly- reciprocally-continuous nature of the pair $(B, T)$, we find a sequence $\left\{y_{n}\right\}$ in X such that $\lim _{n \rightarrow \infty} B y_{n}=B w$ whenever $\lim _{n \rightarrow \infty} B y_{n}=\lim _{n \rightarrow \infty} \operatorname{Ty}_{n}=$ $\mathrm{w}$ for some $\mathrm{w} \in \mathrm{X}$.

Also since the pair $(B, T)$ is B-compatible mapping of type-(E) then there exists some $w \in X$ such that $\lim _{n \rightarrow \infty} B B y_{n}=\lim _{n \rightarrow \infty} B \mathrm{By}_{n}=$ Tw. Then, Bw=Tw and this shows the pair $(\mathrm{B}, \mathrm{T})$ may have $\mathrm{w}$ as a coincident point.

Now we claim that $A t=B w$.

Put $\mathrm{x}=\mathrm{t}, \mathrm{y}=\mathrm{w}$ in the inequality (iii) of Theorem 3.2, we get

$\mathrm{d}^{*}(\mathrm{At}, \mathrm{Bw}) \leq\left[\mathrm{d}^{*}(\mathrm{St}, \mathrm{Tw}) \mathrm{d}^{*}(\mathrm{At}, \mathrm{St}) \mathrm{d}^{*}(\mathrm{Bw}, \mathrm{Tw}) \mathrm{d}^{*}(\mathrm{St}, \mathrm{Bw}) \mathrm{d}^{*}(\mathrm{At}, \mathrm{Bw})\right]^{\frac{\lambda}{6}}$

$d^{*}(A t, B w) \leq\left[d^{*}(A t, B w) d^{*}(A t, A t) d^{*}(B w, B w) d^{*}(A t, B w) d^{*}(A t, B w)\right]^{\frac{\lambda}{6}}$

$\mathrm{d}^{*}(A t, B w) \leq\left[\mathrm{d}^{*}(A t, B w) \mathrm{d}^{*}(A t, A t) \mathrm{d}^{*}(B w, B w) \mathrm{d}^{*}(A t, B w) \mathrm{d}^{*}(A t, B w)\right]^{\frac{\lambda}{6}}$

$\mathrm{d}^{*}(A t, B w) \leq\left[\mathrm{d}^{*}(A t, B w)(1)(1) \mathrm{d}^{*}(A t, B w) \mathrm{d}^{*}(A t, B w)\right]^{\frac{\lambda}{6}}$

$\mathrm{d}^{*}(\mathrm{At}, \mathrm{Bw}) \leq\left[\mathrm{d}^{* 3}(\mathrm{At}, \mathrm{Bw})\right]^{\frac{\lambda}{6}}$

$\mathrm{d}^{*}(\mathrm{At}, \mathrm{Bw}) \leq\left[\mathrm{d}^{*}(\text { At, Bw })\right]^{\frac{\lambda}{2}}$ this implies At $=\mathrm{Bw}$.

Next we shall claim that $t=w$.

Put $\mathrm{x}=\mathrm{x}_{\mathrm{n}}$ and $\mathrm{y}=\mathrm{y}_{\mathrm{n}}$ in the inequality (iii) of Theorem 3.2 then

$d^{*}\left(A x_{n}, B y_{n}\right) \leq\left[d^{*}\left(S x_{n}, T y_{n}\right) d^{*}\left(A x_{n}, S x_{n}\right) d^{*}\left(B y_{n}, T y_{n}\right) d^{*}\left(S x_{n}, B y_{n}\right) d^{*}\left(A x_{n}, B y_{n}\right)\right]^{\frac{\lambda}{6}}$

letting $\mathrm{n} \rightarrow \infty$

$d^{*}(t, w) \leq\left[d^{*}(t, w)\right]^{\frac{\lambda}{2}}$

this implies $t=w$.

Therefore $\mathrm{At}=\mathrm{St}=\mathrm{Bt}=\mathrm{Tt}=\mathrm{t}$, this gives $\mathrm{t}$ is a common fixed point of self-maps $\mathrm{A}, \mathrm{B}, \mathrm{S}$ and $\mathrm{T}$.

To prove the uniqueness, let $q$ be another common fixed point. Then on putting $x=t$ and $\mathrm{y}=\mathrm{q}$ in the inequality (iii) of Theorem 3.2 gives

$d^{*}(t, q)=d^{*}(A t, B q) \leq\left[d^{*}(S t, T q) d^{*}(A t, S t) d^{*}(B q, T q) d^{*}(S t, B q) d^{*}(A t, B q)\right]^{\frac{\lambda}{6}}$

$d^{*}(t, q) \leq\left[d^{*}(t, q) d^{*}(t, t) d^{*}(q, q) d^{*}(t, q) d^{*}(t, q)\right]^{\frac{\lambda}{6}}$

$d^{*}(t, q) \leq\left[d^{*}(t, q)\right]^{\frac{\lambda}{2}}$

this implies $\mathrm{t}=\mathrm{q}$.

Hence, the self-maps A, B, S and T have the unique common fixed point.

Finally we prove another common fixed point theorem using different conditions.

Theorem 3.3: Let A, B, S, T: $X \rightarrow X$ be four self-maps of multiplicative symmetric space $\left(\mathrm{X}, \mathrm{d}^{*}\right)$ such that 
(i) one of the pairs $(A, S)$ or $(B, T)$ is compatible of type-E and reciprocally-continuous

(ii) $\mathrm{A}(\mathrm{X}) \subseteq \mathrm{T}(\mathrm{X})$ or $\mathrm{B}(\mathrm{X}) \subseteq \mathrm{S}(\mathrm{X})$

(iii) one of the a pairs $(\mathrm{A}, \mathrm{S})$ or $(\mathrm{B}, \mathrm{T})$ is weakly-compatible

(iv) $d^{*}(A x, B y) \leq\left[d^{*}(S x, T y) d^{*}(A x, S x) d^{*}(B y, T y) d^{*}(S x, B y) d^{*}(A x, B y)\right]^{\frac{\lambda}{6}}$

for all $\mathrm{x}, \mathrm{y} \in \mathrm{X}$ where $\lambda \in\left(0, \frac{1}{2}\right)$

Further

(iv) The sequence $\left\{B y_{n}\right\}$ converges $\forall\left\{y_{n}\right\}$ in $X$ whenever $\left\{\mathrm{Ty}_{n}\right\}$ converges or the sequence $\left\{\mathrm{Ax}_{\mathrm{n}}\right\}$ converges $\forall\left\{\mathrm{x}_{\mathrm{n}}\right\}$ in $\mathrm{X}$ whenever $\left\{\mathrm{Sx}_{\mathrm{n}}\right\}$ converges.

Then, the self-maps $A, B, S$ and $T$ have the unique common fixed point.

Proof: If pair of the mappings $(A, S)$ is reciprocally-continuous and compatible mapping of type-(E) then, there exists a sequence $\left\{x_{n}\right\}$ in $X$ satisfy $\lim _{n \rightarrow \infty} A x_{n}=\lim _{n \rightarrow \infty} S x_{n}=z$ for some $\mathrm{z} \in \mathrm{X}$ and this gives $\mathrm{Az}=\mathrm{Sz}$. The condition $\mathrm{A}(\mathrm{X}) \subseteq \mathrm{T}(\mathrm{X})$ implies that there we find a sequence $\left\{y_{n}\right\}$ in $X$ such that $\lim _{n \rightarrow \infty} A x_{n}=\lim _{n \rightarrow \infty} \operatorname{Ty}_{n}=z$.

We claim that $\lim _{n \rightarrow \infty} B y_{n}=z$ if not, substitute $x=x_{n}$ and $y=y_{n}$ in the inequality (iv) of Theorem 3.3

$d^{*}\left(A x_{n}, B y_{n}\right) \leq\left[d^{*}\left(S x_{n}, T y_{n}\right) d^{*}\left(A x_{n}, S x_{n}\right) d^{*}\left(B y_{n}, T y_{n}\right) d^{*}\left(S x_{n}, B y_{n}\right) d^{*}\left(A x_{n}, B y_{n}\right)\right]^{\frac{\lambda}{6}}$

letting $\mathrm{n} \rightarrow \infty$

$\mathrm{d}^{*}\left(\mathrm{z}, \mathrm{By}_{\mathrm{n}}\right) \leq\left[\mathrm{d}^{*}\left(\mathrm{z}, \mathrm{By_{n }}\right)\right]^{\frac{\lambda}{2}}$ a contradiction .

This implies $\lim _{n \rightarrow \infty} B y_{n}=z$

We claim that $\mathrm{z}=\mathrm{Az}=\mathrm{Sz}$, if not by putting $\mathrm{x}=\mathrm{z}, \mathrm{y}=\mathrm{y}_{\mathrm{n}}$ in the inequality (iv) of Theorem 3.3

$d^{*}\left(A z, B y_{n}\right) \leq\left[d^{*}\left(S z, T y_{n}\right) d^{*}(A z, S z)\left(B y_{n}, T y_{n}\right) d^{*}\left(S z, B y_{n}\right) d^{*}\left(A z, B y_{n}\right)\right]^{\frac{\lambda}{6}}$

letting $\mathrm{n} \rightarrow \infty$

$\mathrm{d}^{*}(\mathrm{Az}, \mathrm{z}) \leq\left[\mathrm{d}^{*}(\mathrm{Az}, \mathrm{z})\right]^{\frac{\lambda}{2}}$

this implies $\mathrm{Az}=\mathrm{z}$.

This shows $\mathrm{Z}$ is a common fixed point for the mappings $\mathrm{A}$ and $\mathrm{S}$.

Since $A(X) \subseteq T(X)$ means we get a point $w \in X$ such that $z=A z=T w$.

We will show that $\mathrm{Tw}=\mathrm{Bw}$, by taking $\mathrm{x}=\mathrm{z}$ and $\mathrm{y}=\mathrm{w}=\mathrm{z}$ in inequality (iv) of Theorem 3.3 then we have,

$\mathrm{d}^{*}(\mathrm{Az}, \mathrm{Bw}) \leq\left[\mathrm{d}^{*}(\mathrm{Sz}, \mathrm{Tw}) \mathrm{d}^{*}(\mathrm{Az}, \mathrm{Sz}) \mathrm{d}^{*}(\mathrm{Bw}, \mathrm{Tw}) \mathrm{d}^{*}(\mathrm{Sz}, \mathrm{Bw}) \mathrm{d}^{*}(\mathrm{Az}, \mathrm{Bw})\right]^{\frac{\lambda}{6}}$

$\mathrm{d}^{*}(\mathrm{z}, \mathrm{Bw}) \leq\left[\mathrm{d}^{*}(\mathrm{z}, \mathrm{z}) \mathrm{d}^{*}(\mathrm{z}, \mathrm{z}) \mathrm{d}^{*}(\mathrm{Bw}, \mathrm{z}) \mathrm{d}^{*}(\mathrm{z}, \mathrm{Bw}) \mathrm{d}^{*}(\mathrm{z}, \mathrm{Bw})\right]^{\frac{\lambda}{6}}$

$\mathrm{d}^{*}(\mathrm{z}, \mathrm{Bw}) \leq\left[\mathrm{d}^{*}(\mathrm{z}, \mathrm{Bw})\right]^{\frac{\lambda}{2}}$

which is not possible this implies $\mathrm{z}=\mathrm{Bw}$ and this gives $\mathrm{Bw}=\mathrm{Tw}=\mathrm{z}$.

Now we use weakly compatible nature of the pair $(B, T)$ implies $z=B z=T z$ and consequently $\mathrm{Az}=\mathrm{Bz}=\mathrm{Sz}=\mathrm{Tz}=\mathrm{z}$. This implies $\mathrm{z}$ is a common fixed point of the selfmappings $A, B, S$ and T. Further the uniqueness of common fixed point can be easily verified. 


\section{Conclusion}

In this paper three common fixed point theorems are established. In the first result, two pairs are assumed to be weakly reciprocally continuous and compatible-E. In the second theorem, the notion of compatible mappings of type-E and reciprocally-continuous mappings are used in splitting form. Finally in the last theorem the notion of one of the pairs of compatible mappings of type-E along with reciprocally continuous mappings and the other pair as weakly compatible mappings with convergence is used.

\section{References}

1. M. Grossman and R. Katz, Non-Newtonian Calculus (Lee Press, Pigeon Cove, MA, 1972).

2. A. E. Bashirov, E. M. Kurplnara, and A. Ozyaplcl, J. Math. Anal. Appl. 337, 36 (2008). https://doi.org/10.1016/j.jmaa.2007.03.081

3. M. Ozavsar and A. C. Cevikel, Appl. Math. 1-14 (2012).

4. K. Menger, Math. Annalen 100, 75 (1928). https://doi.org/10.1007/BF01448840

5. M. Cicchese, Boll. Un. Math. Ital. 13- A(5), 175 (1976).

6. G. Jungck, Int. J. Math. Math. Sci. 11, 285 (1988). https://doi.org/10.1155/S0161171288000341

7. M. R. Singh and Y. M. Singh, Hacettepe J. Math. Statist. 40, 503 (2011). https://dergipark.org.tr/en/pub/hujms/issue/7759/101418

8. R. P. Pant, Ind. J. Pure. Math. 30, 147 (1999).

9. V. Srinivas, B .V. B. Reddy, and R. U. Rao, Int. J. Theor. Appl. Sci. 5, 154 (2013).

10. A. Tomar, S. Upadhyay, and R. Sharma, 7, 116 (2019).

11. S. Beloul and A. H. Ansari, Bull. Int. Mathematical Virtual Inst. 8, 345 (2018). 